\title{
IMPACTED AND UNERUPTED CUSPIDS AND THIRD MOLARS*
}

\author{
By Dr. Allen E. Scott, San Francisco, Cal.
}

\begin{abstract}
IN young patients with whom we most frequently concern ourselves, there $\boldsymbol{I}$ is often considerable doubt as to whether a tooth is impacted or simply has not erupted. In this paper it is assumed that an impacted tooth is one that will not or cannot erupt in the course of time, while an unerupted tooth is one that will erupt either in or out of its normal position if sufficient time is allowed to elapse. This statement applies, of course, to dentures where no orthodontic treatment has been given. When space is produced for an impacted tooth it may then become an unerupted tooth. While it usually happens that premolars will erupt when sufficient space is provided, the same statement does not hold true of eanines.
\end{abstract}

There is no doubt that the human denture is undergoing a change. This is probably due to the nature of our food as well as the mode of taking it. There seems to be a great tendency toward the elimination of the lateral incisors; the absence of a premolar is by no means an uncommon occurrence.

Impacted teeth usually result in considerable discomfort to both patient and dentist. If let alone they are apt to cause considerable pain by pressure on nerves; they may result in the formation of a cyst; or in the event that they are left when other teeth are extracted, they sometimes erupt under a plate or bridge. When this occurs, their removal is usually necessary and the construction of a new plate or bridge is usually indicated. From this it is quite evident that an impacted or unerupted tooth should receive our attention.

We are probably called on to deal with impacted maxillary canines and mandibular third molars more than any other teeth in the mouth. The treatment of these conditions is very different. In the case of the eanines it is usually desirable to move the tooth into its proper position, while in the case of third molars, with the exceptions as stated later on in this paper, it is desirable to remove the tooth. Due to the position of the third molar, it is a very hard tooth to keep clean and therefore is usually subject to an early attack of earies. When it happens that the second molars are badly decayed or have been devitalized, it is often considered best to advise the extraction of the second molars and allow the third molar to come forward into the space created.

It is rather difficult to draw a distinct line of demarcation between the impacted canines that ean be moved into position and those that have to be

${ }^{*}$ Read before the Pacific Coast Society of Orthodontists, Portland, Oregon, Feb. 16-18, 1921. 
extracted. But in general the treatment is dependent on about three conditions:

1. The position of the tooth or the distance to be moved.

2. The age of the patient.

3 . The condition of the teeth in general.

In some cases the canine is so high in the substance of the maxillary bone that it is an extremely difficult operation to successfully place a pin in the tooth. The determination of this should, of course, be left to the judgment of the surgeon. Without very much data to substantiate the statement, I think that it is feasible to move into position most any canine in which it is possible to place a pin for the attachment of an appliance.

In these cases the Mershon appliance is used. The base wire is bent in such a way as to allow the canine to come into position. An auxiliary spring is soldered to the base wire in the molar region. This is attached to the pin by means of a piece of wire ligature and the patient dismissed for about a month. The tooth usually moves very slowly at first, due to the simple fact that both crown and root are in bone substance. But when the crown has been moved into soft tissue, movement takes place at the ordinary rate that we are accustomed to move teeth. When the crown of the tooth is sufficiently exposed, the pin which has been placed by the surgeon is removed if possible. If it is not possible to remove the pin, it is cut off and a band placed on the tooth. From this point on, the same treatment is followed as would be used when a tooth is in ordinary malposition. In operating on these cases Dr. James G. Sharp uses a threaded wire the same size as the drill. By following this technic the pin is securely placed and very little trouble is experienced by the pin's being loosened and coming out.

From our present knowledge mandibular third molars seem to be impacted in increasing numbers. This is in all probability due to a tendency of the human race to use the jaws less and less for mastication as civilization advances. There seems to be no doubt that a great many of our dental defects are due to our mode of living under our present state of advancement. It appears that nature does not develop enough bone to properly accommodate the third molar.

In young patients an unerupted third molar is apt to be taken for one that is impacted. In patients ten or twelve years of age these teeth usually slant somewhat to the mesial when they are occupying a normal position. It has been shown in numerous cases that mandibular impacted molars may cause the anterior mandibular teeth to rotate and lap. In these cases, of course, their surgical removal is indicated. However, where it is desired to obtain forward movement or development of the mandible it is not desirable to remove these teeth until late in the period of treatment. With a retaining appliance in place so as to prevent crowding of the anterior teeth the pressure of the impacted third molar will be exerted in such a way as to crowd all the mandibular teeth forward. In this connection it is well to remember that the longer the teeth are left in position, the more developed the roots become and their removal is rendered more difficult and complicated. 


\section{DISCUSSION}

Dr. W. R. Dingham, Portland, Oregon.-I have gone over Dr. Scott's paper and agree with him on practically all of his points. The movement of impacted canines is accomplished in several ways, but I think the method wherein the lingual appliance is used is the best as from it you can get practically any direction of force that may be required. Very often the impacted canine cases are in older patients and for the purpose of maintaining the space and giving a better appearance, a canine facing can be soldered to the lingual appliance and the space thus maintained and the appearance improved while the impacted canine is being brought down. This form of appliance ean also be used in impacted incisors. Where you have soldered a facing on the lingual appliance, in the case of impacted teeth, the lingual appliance and facing also serve a valuable purpose in giving you the relations of the teeth and parts when taking an x-ray. By taking a picture through the top of the skull so as to give an ocelusal view of the teeth and appliance, the impacted tooth can be easily located, if pictures of the other usual angles have been taken. Before the use of the $x$-ray, we cut into the tissue, tried to locate the tooth, and very often we missed it. I was timid about cutting away very much of the process at the time $I$ was trying to locate these teeth. It was my practice to make a very small opening and try to put a pin into the tooth, feeling sure where it was going and the direction of the tooth. Now with the aid of the x-ray we ean go in and open the entire area and expose the erown of the tooth and determine positively the direction of the tooth. By removing a good portion of the bone from in front of the crown, the resistance to the tooth movement is lessened and the tooth responds more rapidly. There are several different methods of attaching to the impacted teeth. Dr. Dunn has one I have never seen, before. Some men are able to put a band on a tooth even when it is deeply imbedded in the process. Dr. Engstrom explains how he gets a band on a tooth and cements it in position, which plan does not injure the tooth as does placing a pin in the erown.

There is no question but that the impacted canines cause much trouble from pressure, thereby producing reflex nervous conditions. They also cause absorption of the roots of the other permanent teeth. I recall one case similar to the one Dr. Seott speaks of in which the complete absorption of the lateral incisor root was produced by an impacted canine. The question of the age of the patient is an important feature in this work. The older the patient, the greater the difficulty in bringing down these impacted teeth.

Dr. C. O. Engstrom, Sacramento, Calif.-The procedure I have used for many years is about the same as that explained by Dr. Scott. However, I use a cap instead of a band, as the doctor has just stated. My idea is not to mutilate the tooth at all. The cap can be cemented to the surface of the tooth, and sufficient pressure may be exerted to move the tooth. In one case I treated, it was necessary to place a cap on a canine, which was located, it seemed to me, fully a half inch within the outward plate of the superior maxillary bone. It was a considerable distance. I cemented a cap on the tip of the eanine and moved the tooth out suffieiently so that it might be banded. I think the next paper on the program will describe a method very similar to my own.

Dr. William Cavanagh, Portland, Oregon.-I fear we may be guilty of perpetrating a crime on the third molar in orthodontic treatment sometimes. If we begin the treatment of a case at the age of six to twelve years, the development of the jaws is forward. I believe when we think we are stimulating the development of the jaws in a certain direction that we are retarding them in another. My opinion is that when we place stationary anchorage on the permanent molars, for a period of years for the purpose of producing lateral development, we are prohibiting a growth of the arches in a forward direction. The third molar, as Dr. Scott stated, has a forward direction in early life and only assumes an upright position when the jaws develop forward and the second molar moves anteriorly and allows space for the third molars to erupt. If we prohibit the forward movement of the third molar we have stopped the normal action and purpose of the inclination of the third molar; namely, to assist the forward development of the arch in keeping with the cranium. So, I fear that we have been stopping development in certain 
cases that would have occurred but for the appliances that were used. I will ask whether any of you have ever heard a theory as to why we get these abnormal inclinations of these canines? There are so many cases in which the cusp of that tooth is almost at a level with the root. We have other theories as to why other malpositions occur, but I have never seen any reason why the canine should assume such a perverted position.

Dr. Chas. C. Mann, Seattle, Wash.-I remember one case where the x-ray pictures showed the canine apparently coming down into correct position. The deciduous canine was in position, and the arch was approximally normal. The patient did not want the deciduous tooth extracted because of appearanee; so we left it, depending upon the x-ray for assurance that the deciduous tooth would absorb and the permanent tooth come into the arch. Neither one of those things occurred. Later we made another pieture, in three or four months, and the root of the deciduous tooth had deflected the crown of the permanent tooth and it was lying almost horizontally across the other tooth. It eventually passed the median line and was extracted. I believe the extreme inclination of the canines is due to a general lack of development of the arch. Being the last teeth to erupt, they are easily deflected from their course. My experience with an impacted central may be of some interest. In one case the oral surgeon made an incision and removed the process and I started the movement of the tooth. However, so much bone had been removed that it never regenerated. Although the tooth is in position it seems hopeless, as the bone has never developed around. it. So I would caution you in regard to removing too much bone in the intermaxillary region.

Dr. H. L. Morehouse, Spokane, Wash.-I would like to answer Dr. Cavanagh's statement with regard to the impacted third molar. As you know, the impacted third molar is my hobby. I think I have stated before that in many of our Class II cases, with prominent canines, or what is termed receding ineisors, that condition has been brought about to a more or less degree by the natural, as Dr. Cavanagh explains it, development due to the force of the erupting second and third molars. I say this from experience with my own daughter's teeth. I treated her teeth at five and one-half years of age, making the necessary expansion for the eruption of the permanent set. I was careless in watching the mouth at the time of the eruption of the second molar. The eruption of the second molar forced mesially the entire line of teeth, premolars and canines to a minor degree. After that, I made radiograms of the third molars, and found she had both maxillary and mandibular third molars impacted at fourteen years of age. I feel that there is possibly a very slight chance of checking that mesial development that is expected, or which we used to be taught is to be expected from the mesial inclination of the second or third molars. If you will make $x$-ray pictures of your patients' jaws from six years right along, if you cannot tell at the time you first find the germ of the third molar showing, that you will have an impacted third molar, then I am very much mistaken. If the tooth is going to be impacted, it will show at the commencement of development of the tooth germ. It follows along the same line as the

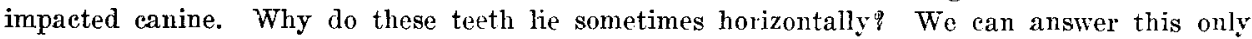
in one way to the patients. The germ is deposited on that angle, in that direction, and that is the direction in which the tooth will erupt. It is a slip in nature, to the same extent that the cleft palate in the majority of instances is a slip. Cleft palate is not always due to disease. It may be a threatened miscarriage, or something of that kind. So we have the same thing in the malposition of the canine teeth, $\mathrm{I}$ think; but with the third molar, while there is a chance if you will allow the distal movement of the second molar, you may get added impaction, and, as I said in a paper a year ago, the impaction may be due to opening up spaces for upper premolars.

Dr. P. T. Meaney, Portland, Oregon.-The abnormal positions of the canines (especially the maxillary) are due, I believe, to a lack of length in the arch, mesio-distally. The lack of length is the result of premature extraction, or loss of tooth structure by decay, of the deciduous canines, also first and second molar.

As early as the third or fourth year of the child's life, the first permanent molar is exerting a force which causes the first and second deciduous molars, also canines to move toward the point of least resistance which is mesially, thereby lengthening the arch. 
If the mesio-distal diameter of the deciduous molars and canines is destroyed by caries or these teeth are lost by extraction, the mesial movement of the first permanent molar is the result, occupying a position mesial to the normal, approximately the width of the lost tooth or tooth structure and more or less so in the mouths of youngsters under eight years old; after that age the premolars are a resistance to mesial movement of the first permanent molars.

The above lack of length of the arch will not accommodate the permanent canine, therefore it is deflected labially or lingually to its normal position.

The position the canine maintains in the arch is a solution for its easy displacement, where it has not sufficient room for normal eruption.

The fact that the carine is a penetrative and prehensive organ is also a reason why it assumes many abnormal positions ir the arch when forced to erupt in arches not developed normally.

Dr. E. C. Reed, Long Beach, Calif.-I would like to ask, since we are speaking so much with reference to the forward movement due to the eruption of the third molar, is there not a change in the angle of the mandible, changing from a more or less obtuse, to more nearly a right angle, thus providing a posterior development of the jaw, giving additional room for the accommodation of the teeth?

Dr. Charles C. Mann, Seattle, Wash.-I wish to take issue with the president's theory that the misplaced canine is the result of misplacement of the germ of the tooth at the time of its formation, as it is laid down in the membrane of the individual.

Dr. Morehouse.-I referred primarily to the horizontal position in which the canine teeth are found, and not to minor deflections.

Dr, Mann,-Possibly I misunderstood you, but the fact remains that if we aceept a theory of that sort we must set aside many other things we have been led to believe by experience of the past, as contributing to the normal development of the arches. These things are related one with the other. So, if we are to acept the theory that these things are prearranged, we might as well discard the other forces which we think play a part in the development of the jaws. I feel as Dr. Reed does, whether we care to accept it or not, that there must be some posterior development due to a change in the angle of the mandible; there must be some compensatory development there that increases the space for the third molars in these cases. I believe it might be well to go a little more slowly in our treatment and ascertain by the use of the $x$-ray whether these third molars have been impacted prior to our treatment, or whether by hurrying the treatment in the anterior region of the arch and exerting distal pressure we did not tend to impact the third molar that had originally only a slight inclination.

By the continued use of the $x$-ray, we can determine fairly well the possibilities of the third molar coming into correct position. I have observed where you have the mesial marginal ridge of the third molar caught gingivally to the contact of the second molar that the third molar will not come into normal position, but if the mesial marginal ridge of the third molar is occlusal to the contact point of the second molar and there is no backward pressure on the second molar, the third molar will assume a normal position. By using the second deciduous molars for anchorage in as many cases as possible, we are less liable to displace the first permanent molars distally.

Dr. Morehouse.-I want to make myself clear as to the cuspids. I think the theory that I advanced with regard to the canine that was impacted to the degree of being horizontally placed with the cusp lying at right angles to the root of the centrals-

Dr. Mann.-Did you have an opportunity to observe it at the beginning of the formation of the canines

Dr. Morehouse.-When the root was one-half or two-thirds formed. We sometimes find a supernumerary germ completely reversed in its root direction. Here the same theory would apply. I have x-ray pictures of a maxillary supernumerary tooth germ that lay with its root.headed downward instead of upward. We had one the other day, a completely 
formed lateral incisor, a supernumerary, with the root pointing where the erown should be. On the same theory the germ was laid down that way and it will grow that way in spite of anything.

Dr. Mann.-We, as orthodontists and general dentists have not had as much experience and study as we need. I do not think a rare exception would prove the average rule.

Dr. W. R. Dingham, Portland, Oregon.-In what percentage of cases do we have an impaction of the third molars at an early age? I have difficulty in determining when a third molar is impacted in a young patient.

Dr. Morehouse.-I have not had the time this year to do the $\mathbf{x}$-ray work on younger patients which I had hoped to do. I never start a case of malocelusion ten to twelve years of age without having x-rays for third molars, especially if any outward tendency toward impaction is in evidence. It would be very interesting, and $I$ would suggest that some members who have a little time would start in at six and follow up a few eases that indicate the possibility of impaction, following them from year to year, and see how they develop. I am positive the third molar is misplaced at an early age. I am going to prove it some day, but I have not had time this year.

Dr. R. S. Baldwin, Spokane, Wass.--In the line of hypothetical theorizing on causes of canine displacement you may assume that the pressure of the erupting tooth causes absorption of the deciduous tooth. Now in most eases that we see, the incoming canines have a tendeney, if there is a crowded condition, to erupt labially or lingually to the deciduous canine. This might be on account of the conical shape of the permanent canine which gives very little contact surface to exert the influence for disintegrating the root of the deciduous canine. And if that incoming tooth does not strike the deciduous root with sufficient contact to cause disintegration, it passes that root by, and starts in a deflected course. In many cases where you have impacted canines, the deciduous canine roots exhibit practically no disintegration, and the permanent canines lie laterally to the vertical direction of the deciduous root. The canines are peculiar in the manner of eruption and in the way the deciduous roots are sometimes retained, and the ease with which the retained roots deflect the permanent tooth. If, as the result of an early x-ray diagnosis, we would extract the deciduous canine it would aid materially in correcting that condition before the permanent tooth is deflected beyond hope.

Dr. Scott. - The whole thing reminds me of the conversation with a man who has to do with a weather bureau. He says "It will rain tomorrow," "How do you know q", "The barometer is going down." "Why." "Because the air pressure is going down." And that is as far as we get. These teeth are out of normal position because the germ is misplaced. Why is the germ misplaced We do not bring our argument to a successful conclusion. We do not get anywhere. Since the normal position of the canine is between the lateral and the first premolar it may be during the forward development of the arch these teeth move forward, and the canine being placed high in the bone, does not come under that forward influence and is left behind in the development, and when it does erupt it erupts out of its normal position.

It is certainly more desirable to place a cap or some sort of a band on a tooth, such as Dr. Engstron and Dr. Dunn have referred to. You cannot always tell exactly the position of the crown. Some years ago a man operated for Dr. Suggett, putting a pin in the root of a tooth, instead of in the crown. The third molar has become more and more interesting as we have studied it, and our studies on it are not exhausted by any means. We have to reckon with this tooth more and more as we proceed. It occurs to me the reason for some of these things may not be so particularly necessary unless they lead to the removal of their effect. One more thought, as to the age for the removal of these third molars. In our office, we sometimes leave them in some little time, but you must remember the roots are being developed all the while, and the longer they remain, the more difficulty one may have in their removal. 